\title{
Adecuación del grado de desarrollo de la formación en competencias a la necesidad en el entorno laboral, según la opinión de los estudiantes
}

\section{The adequacy of the degree of development of the formation in competitions to the need in the job environment, according to the opinion of the students}

\author{
$\mathrm{M}^{\mathrm{a}}$ Covadonga de la IGLESIA VILLASOL \\ Universidad Complutense de Madrid
}

Recibido: Junio 2010

Aceptado: Marzo 2011

\section{Resumen}

En este trabajo se ofrece un estudio descriptivo de los resultados de la encuesta realizada a los estudiantes que realizan el cuarto y quinto curso de las licenciaturas de Economía y Administración de Empresas, en las Facultades de Ciencias Económicas y Empresariales de la Universidad Complutense de Madrid y de la Universidad de Alcalá de Henares, sobre la percepción que ellos tienen de su propio grado de desarrollo competencial y la valoración que hacen de lo relevante que creen que el mismo puede ser en el entorno laboral. La información está segmentada según que el estudiante tenga o no una experiencia profesional previa, el sexo, la especialidad que cursa, la licenciatura o el curso en el que está matriculado.

Palabras claves: Competencias, formación, valoración, inserción laboral.

\begin{abstract}
In this paper we offers a descriptive study of the results to the survey of students who were in the fourth and fifth year of the Bachelor of Economics and Business Administration in the Faculty of Economics and Business Administration at the Universidad Complutense de Madrid and University of Alcalá de Henares, about the perception they have their own level of skills development and the assessment of how relevant they believe the same may be at work. The information is segmented according to whether or not the student has previous professional experience, sex, the specialty courses, degree or course in which it is registered.
\end{abstract}

Key words: Skills, formation, valuation, labor insertion

Todos los claustros universitarios tienen como reto aportar a la sociedad una enseñanza superior de calidad y unos egresados altamente cualificados, para lo cual desarrollan todo tipo de procesos de evaluación, internos y externos, que les permita tener un mejor conocimiento de la efectividad docente. Este proceso de mejora continua debe ir dirigido, principalmente, al desarrollo de contenidos, actividades 
formativas y recursos docentes que favorezcan que los estudiantes, durante su proceso formativo, no solo desarrollen y asimilen los conocimientos propios de su titulación, sino también las competencias específicas y las habilidades transversales que demanda su entorno profesional, cambiante y cada vez más globalizado, como una medida adicional del éxito formativo de las Universidades.

Este trabajo se nutre de la información estadística elaborada en el Proyecto de Innovación y Mejora de la calidad Docente, "Plan de Mejora docente de la formación en competencias genéricas de los estudiantes. De la Universidad al entorno laboral" ${ }^{1}$, cuyo objetivo es proporcionar una información comparativa sobre la percepción que los estudiantes tienen de su grado de desarrollo competencial y la valoración que hacen de lo relevante que es el mismo en el entorno laboral, según sus vivencias previas. Para abordar este trabajo se realizó un proceso de encuestación a los estudiantes de cuarto y quinto curso de las Facultades de Ciencias Económicas y Empresariales de las Universidades Complutense de Madrid y Alcalá de Henares.

La primera explotación de los datos recoge un análisis descriptivo exhaustivo, cuyo resumen se puede consultar en Iglesia e Ionita (2010), y pretende abrir el debate a la opinión de los distintos actores ${ }^{2}$ del proceso formativo sobre la asimetría observada entre la percepción que los estudiantes tienen de los requerimientos en el mercado laboral de egresados con un fuerte desarrollo de competencias genéricas, tanto instrumentales, como sistémicas y personales/emocionales, y el grado en el que éstas se desarrollan durante la etapa formativa universitaria.

\section{Motivación y objetivo de la Investigación}

La motivación de esta línea de trabajo, analizar la percepción de los estudiantes sobre su grado de desarrollo competencial frente a la valoración de la importancia en el entorno laboral, parte de trabajos previos ${ }^{3}$ en los que, y como ya es habitual en los estudios de inserción laboral, al evaluar distintos ítems de la satisfacción de los egresados con el proceso formativo, se observa una clara asimetría tanto en la

${ }^{1}$ Financiado por el Vicerrectorado de Desarrollo y Calidad Docente de la UCM. El equipo de investigación está integrado por los profesores Galleo, E., Gracia, E., Iriondo, I. y De la Iglesia, M.C., siendo ésta la responsable. Se agradece la colaboración extraordinaria de Ionita, C., en la elaboración de informe básico del proyecto.

${ }^{2}$ Actualmente se está complementando la encuesta de opinión de los estudiantes con la obtención de información, cualitativa y cuantitativamente, de responsables de Recursos humanos, el colectivo de docentes y las autoridades académicas.

${ }^{3}$ Véase por ejemplo Iriondo y otros (2009b), que destacan que en general la valoración que hacen los licenciados de la preparación de la Universidad en su desarrollo competencial fue insuficiente en la mayoría de los casos. Los diferenciales en los porcentajes de acumulación de respuestas entre las distintas opciones muestran que la Universidad aportó poco a su preparación, a excepción de "nuevos conocimientos", adecuada en cuanto a los contenidos de los programas teóricos e insuficiente en las áreas de idiomas, informática y capacidad creativa de los licenciados. 
acumulación de respuestas entre las distintas categorías de valoración, como en los valores medios.

El objetivo inicial de este trabajo es contar con un instrumento de efectividad docente, generado con información objetiva del propio proceso educativo, a partir de las opiniones de quienes son los principales protagonistas del mismo, nuestros estudiantes, que oriente sobre cómo se debe replantear la docencia en el contexto de los nuevo planes de estudio, en un momento de cambio metodológico abocado a una orientación hacia el aprendizaje activo, donde se aprenda "haciendo" y, cómo no, desarrollando aquellas habilidades imprescindibles para insertarse laboralmente en un mundo cambiante, claramente competitivo y globalizado. Dicho instrumento proporciona una información comparativa sobre la percepción que los propios estudiantes tienen de su grado de desarrollo competencial y la valoración que hacen de lo relevante que creen que es el mismo en el entorno laboral, según su opinión y vivencias previas.

En los apartados siguientes se aborda en primer lugar la descripción metodológica de la base de datos utilizada y en segundo lugar una visión panorámica y descriptiva de los resultados de la encuesta. En una fase posterior, y no presente en este artículo, se ha desarrollado un análisis factorial que permite extraer o identificar para los diversos ítems o variables un número reducido de factores, constructos o dimensiones subyacentes significativas, a través del estudio de la matriz de covariancia de los datos utilizados de la encuesta.

\section{Metodología de la Investigación}

Los datos de la opinión de los estudiantes utilizada en este trabajo provienen de la encuesta, cuyo cuestionario define las tablas del Anexo, realizada a 444 alumnos en el mes de enero de 2010, matriculados en cuarto o quinto curso ${ }^{4}$ de las licenciaturas de "Administración y Dirección de Empresas" [LADE] o "Economía" [LECO], en las Facultades de CCEE de las Universidades Complutense de Madrid y Alcalá de Henares. El cuestionario, que se corresponde con el utilizado en el Proyecto de Innovación y Mejora de la calidad docente ya citado, recoge un listado exhaustivo de 40 competencias estándares, tratadas como variables independientes, y las preguntas asociadas a las diversas competencias se presentan en dos bloques simétricos con una valoración numérica en escala de Likert de 1 a 5 , según que se refiera a su grado de desarrollo competencial en la formación universitaria (PU) o cómo creen que es de importante en el entorno laboral para el desempeño de la actividad profesional (IT).

\section{Diseño y descripcion de la base de datos}

Señalar que el 49,55\% de los alumnos encuestados son varones, el $42,57 \%$ cursa el quinto año de la licenciatura, el $34,45 \%$ estudia LECO y el $65,31 \%$ declara tener

${ }^{4}$ La selección de los cursos, cuarto o quinto, se realizó dado que los estudiantes ya tienen un criterio más certero del entorno laboral, muchos de ellos están o han realizado prácticas en empresa, o han tenido una experiencia profesional previa. 
experiencia profesional previa. Ahondando en la caracterización de la muestra de encuestados, tabla 1 siguiente, el 47,45\% de los alumnos matriculados de cuarto curso son varones, porcentaje que asciende al 52,23\% en los grupos de quinto curso. Además, el $50,86 \%$ de los que estudian LADE son varones, mientras que entre los de LECO la representación de los mismos baja ligeramente hasta el 47,06\%. Entre los que declaran no tener experiencia laboral previa, el porcentaje de varones es del $55,19 \%$, casi 10 puntos porcentuales superior al $46,55 \%$ de los que declaran tenerla, hecho que muestra un mayor acercamiento al entorno laboral durante su etapa formativa de las féminas frente a sus compañeros varones.

\begin{tabular}{|c|c|c|c|c|c|c|c|c|}
\hline \multicolumn{3}{|c|}{ Curso Cuarto } & \multicolumn{3}{|c|}{ Curso Quinto } & \multicolumn{3}{|c|}{ Total } \\
\hline Hombres & Mujeres & Total & Hombres & Mujeres & Total & Hombres & Mujeres & Total \\
\hline $\begin{array}{c}121 \\
(47,45 \%)\end{array}$ & 134 & $\begin{array}{c}255 \\
{[57,43]}\end{array}$ & $\begin{array}{c}99 \\
(52,23 \%)\end{array}$ & 90 & 189 & \multirow{7}{*}{$\begin{array}{c}220 \\
(49,54 \%)\end{array}$} & \multirow{7}{*}{224} & \multirow{7}{*}{444} \\
\hline \multicolumn{3}{|c|}{ Titulación LADE } & \multicolumn{3}{|c|}{ Titulación LECO } & & & \\
\hline Hombres & Mujeres & Total & Hombres & Mujeres & Total & & & \\
\hline $\begin{array}{c}148 \\
(50,86 \%)\end{array}$ & 143 & $\begin{array}{c}291 \\
{[65,5]}\end{array}$ & $\begin{array}{c}72 \\
(47,06 \%)\end{array}$ & 81 & 153 & & & \\
\hline \multicolumn{3}{|c|}{ SIN experiencia profesional } & \multicolumn{3}{|c|}{ CON experiencia profesional } & & & \\
\hline Hombres & Mujeres & Total & Hombres & Mujeres & Total & & & \\
\hline $\begin{array}{c}85 \\
(55,19 \%) \\
\end{array}$ & 69 & $\begin{array}{c}154 \\
{[34,68]} \\
\end{array}$ & $\begin{array}{c}135 \\
(46,55 \%) \\
\end{array}$ & 155 & 290 & & & \\
\hline
\end{tabular}

Tabla 1: Distribución de la muestra por curso, titulación y experiencia, por sexo y total (\% sobre el total de la segmentación), [\% de la segmentación sobre el total de la muestra]

FUENTE: Encuesta de Necesidad de Desarrollo Competencial. Facultad de CCEE. Elaboración propia

En cuanto a la distribución por tramos de edad de los alumnos encuestados, tabla 2 siguiente, solo el $91,7 \%$ del total de alumnos declara su edad, y mayoritariamente se concentran en el intervalo de 20 a 22 años, con un porcentaje de $48,8 \%$, acumulándose el $89,8 \%$ en edades comprendidas entre 20 y 25 años, mientras que únicamente el $3,19 \%$ de los encuestados encajan en la última categoría, con más de 29 años.

\begin{tabular}{|c|c|c|c|c|c|}
\hline Edad & $\begin{array}{c}\text { Entre 20 y } \\
\text { 22 años }\end{array}$ & $\begin{array}{c}\text { Entre 23 y } \\
\text { 25 años }\end{array}$ & $\begin{array}{c}\text { Entre 26 y } \\
\text { 28 años }\end{array}$ & $\begin{array}{c}\text { De 29 años } \\
\text { 0 más }\end{array}$ & Total \\
\hline Numero & 199 & 167 & 28 & 13 & 407 \\
Porcentaje (\%) & $(48,8 \%)$ & $(41,03 \%)$ & $(6,88 \%)$ & $(3,19 \%)$ & 407 \\
\hline
\end{tabular}

Tabla 2: Distribución de la muestra por tramos de edad y sexo

FUENTE: Encuesta de Necesidad de Desarrollo Competencial. Facultad de CCEE. Elaboración propia

\section{Analisis descriptivo de los resultados}

A continuación se presenta un análisis descriptivo de los resultados de la encuesta de valoración del desarrollo competencial, ordenados por bloques de variables o competencias a efectos de poder presentar los gráficos que ilustran los comentarios. 
Las variables se cuantifican en una escala de Likert con un rango de valores numéricos que van del 1 ("nada") al 5 ("mucho"). El listado completo de competencias analizadas y la información estadística se muestra en las tablas del Anexo.

\section{Primer Bloque de competencias ${ }^{5}$}

El primer bloque de competencias analizadas muestra que en general la opción más representada cuando se pregunta por el grado de desarrollo o de formación en la Universidad es la de "regular", con las excepciones de "comunicación oral y escrita", para la que se acumulan mayoritariamente los alumnos en el escalón de "nada" y las de "organización y planificación" y "búsqueda de información" en el de "bastante". Respecto a la importancia que creen que tienen las distintas competencias en el entorno laboral, la opción más votada es la de "mucho", con porcentajes que en algunos casos superan el 50\% de estudiantes, con las excepciones del "pensamiento analítico y la capacidad de síntesis", la "búsqueda de información en diversas fuentes", el "conocimiento de otras áreas o disciplinas" y "adquirir con rapidez otros conocimientos", que acumulan mayoritariamente a los estudiantes en la opción "bastante".

En cuanto a los valores medios, el gráfico 1 muestra el diferencial en la valoración entre ambas preguntas, pues mientras que para la importancia en el trabajo, en general, es superior a 3,4, llegando a la cota superior de alrededor de 4,3 en la "comunicación oral y escrita en lengua materna", "utilizar herramientas informáticas" y "toma de decisiones", respecto al grado de preparación las medias oscilan entre el 2,1 de "comunicación oral y escrita", y el 3,4 de "comunicación oral y escrita en lengua materna", "pensamiento analítico y capacidad de síntesis", y el 3,3 de "organización y planificación" y "adquirir con rapidez nuevos conocimientos".

Al segmentar la información por submuestras cabe destacar que en general la valoración media de los varones es ligeramente superior a la de sus compañeras las féminas, en todas las competencias cuando se refiere a la importancia en el trabajo, y con excepciones con diferenciales limitados en lo que respecta al grado de preparación en la Universidad, como son "pensamiento analítico y capacidad de síntesis, utilizar herramientas informáticas o búsqueda de información en diversas fuentes". Además, en la segmentación por experiencia profesional, como regla, para el total de alumnos la valoración media del grado de desarrollo o preparación en la Universidad de las competencias es mayor cuando tienen experiencia. Sin embargo, esta unanimidad no se confirma respecto a la importancia en el trabajo.

\footnotetext{
${ }^{5}$ Véase las tablas del anexo para el listado de competencias analizadas
} 


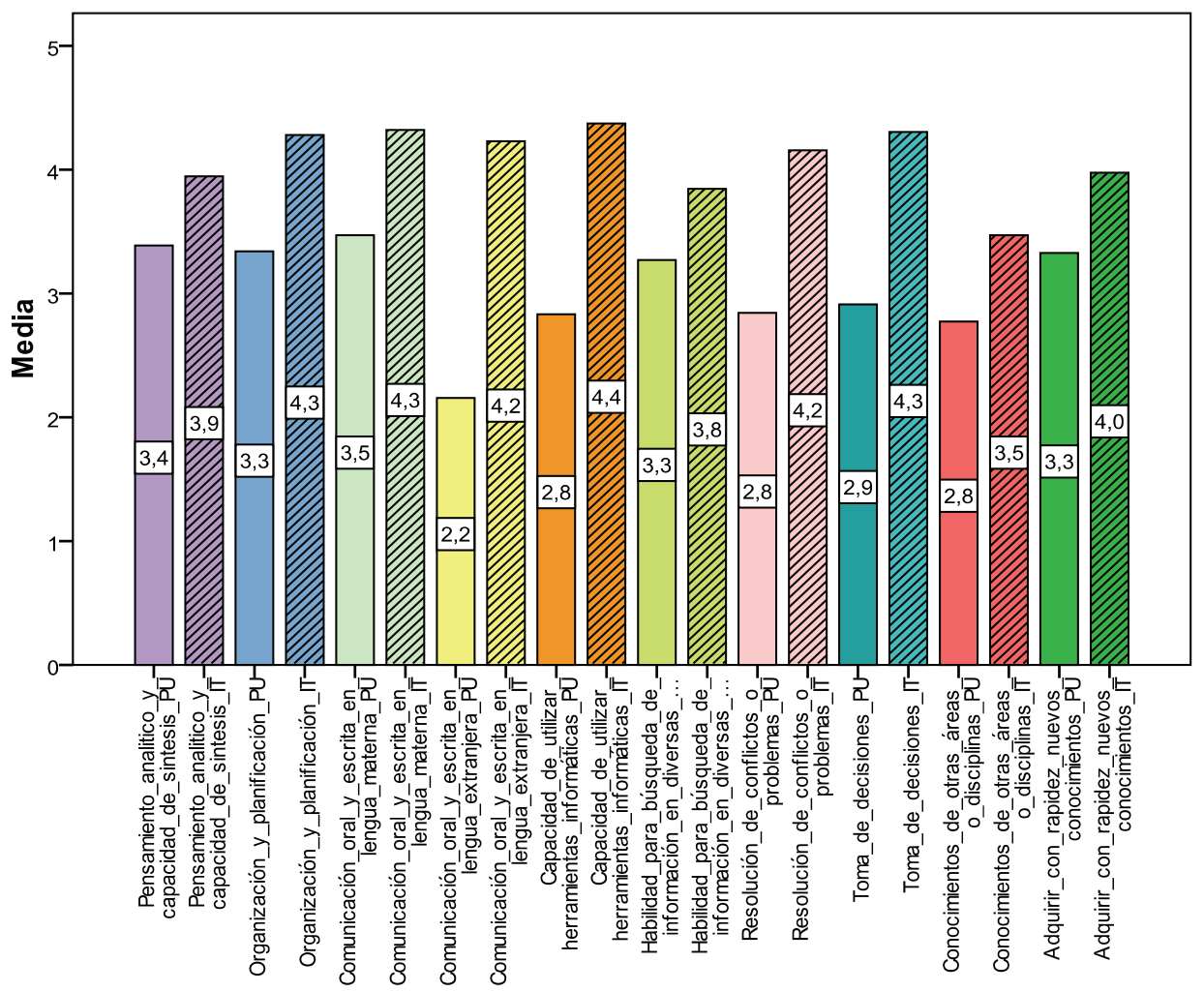

Gráfico 1: Valores medios Primer Bloque de competencias. Preparación en la Universidad (PU) e Importancia en el trabajo (IT) ${ }^{6}$

FUENTE: Encuesta de Necesidad de Desarrollo Competencial. Facultad de CCEE. Elaboración propia

\section{Segundo Bloque de competencias ${ }^{7}$}

Para el segundo bloque de competencias en general no hay unanimidad en la opción más representativa cuando se pregunta por el grado de desarrollo o de formación en la Universidad. Cabe señalar que en las competencias "trabajar en un contexto internacional" y "en entornos diversos y multiculturales", se acumula mayoritariamente a los alumnos en el escalón de "poco", mientras que en las de "trabajar en equipo multidisciplinar" y "rendir bajo presión" y "aprendizaje autónomo", con porcentajes que oscilan del 30 al 39\% de alumnos, se acumulan en el escalón de "bastante". Respecto a la importancia que creen debe tener las distintas competencias en el entorno laboral, en general la opción más votada es la de

${ }^{6}$ En los gráficos 1, 2, 3 y 4, los colores identifican distintas competencias, y para cada color, las barras sin tramas indican la valoración del grado de preparación en la Universidad (PU), y las que tienen trama diagonal indican la importancia en el trabajo (IT).

${ }^{7}$ Véase las tablas del anexo para el listado de competencias analizadas 
"bastante", con porcentajes que oscilan del 36 al 46\% de estudiantes, con excepciones como la "capacidad para hacerte entender", el "trabajo en equipo multidisciplinar", "rendir bajo presión" y el "el uso del tiempo de forma efectiva", que acumulan mayoritariamente a los estudiantes en la opción "mucho" con porcentajes superiores al $43 \%$.

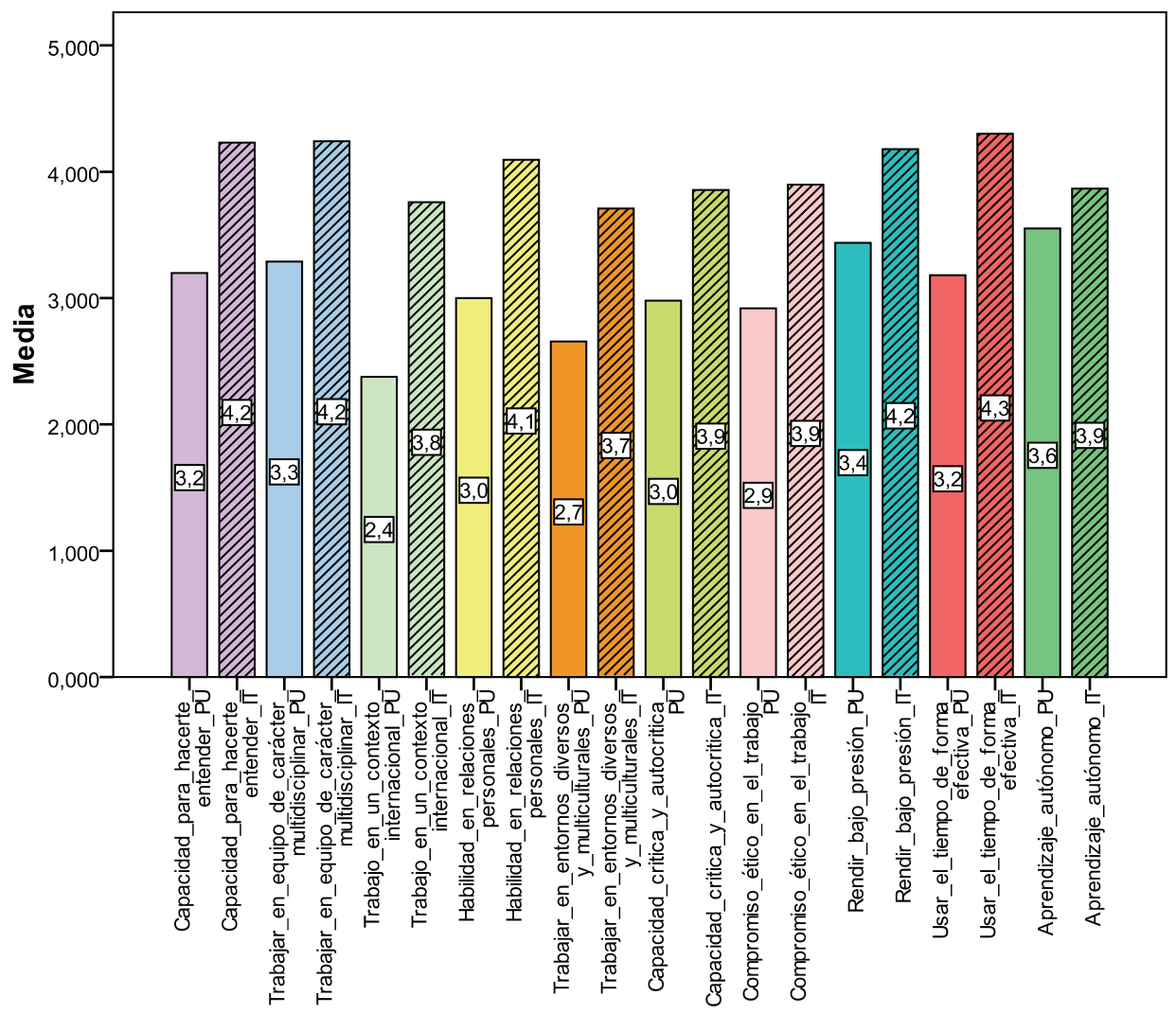

Gráfico 2: Valores medios Segundo Bloque de competencias. Preparación en la Universidad (PU) e Importancia en el trabajo (IT)

FUENTE: Encuesta de Necesidad de Desarrollo Competencial. Facultad de CCEE. Elaboración propia

En cuanto a los valores medios, y respecto a la importancia en el trabajo, en general la media obtenida es superior a 3,7, llegando a la cota superior de 4,3 en el "uso del tiempo de forma efectiva", seguida con más de 4 en la "capacidad para hacerte entender", "trabajo en equipo multidisciplinar", "rendir bajo presión" y "relaciones personales y comunicación interpersonal". Mientras, en el grado de preparación, las medias oscilan entre el 2,3 de "trabajo en un contexto internacional", y el 3,4 de "rendir bajo presión" y el 3,2 de la "capacidad para hacerte entender" y el "trabajo en equipo de carácter multidisciplinar". 
Cabe destacar que en la segmentación realizada por sexo la valoración media de los varones es siempre superior a la de sus compañeras las féminas, en todas las competencias y tanto cuando se refiere a la importancia en el trabajo como al grado de preparación en la Universidad. En la segmentación por experiencia profesional, como regla, para el total de alumnos la valoración media del grado de desarrollo en la Universidad de las competencias es mayor cuando tienen experiencia, y se detecta la misma regularidad respecto a la importancia en el trabajo, a excepción de la competencia "trabajar en un equipo de carácter multidisciplinar".

\section{Tercer Bloque de competencias ${ }^{8}$}

Para el tercer bloque de competencias, en general la opción más representativa cuando se pregunta por el grado de desarrollo o de formación en la Universidad sigue siendo la de "regular", con porcentajes que oscilan entre el 30 y el 39\% de alumnos, con excepciones como "búsqueda de información e investigación" con el 36,04\% de alumnos mayoritariamente en el escalón de "bastante" y "sensibilidad medioambiental" con el $31,53 \%$ en la opción de "nada". Respecto a la importancia que creen que deben tener las distintas competencias en el entorno laboral, en general la opción más votada es la de "bastante", con porcentajes que oscilan del 32 al 48,8\% de estudiantes, con la excepción de "sensibilidad medioambiental", que acumula mayoritariamente a los estudiantes en la opción "regular" con el 30,18\% y "aplicar conocimientos a la práctica", con el 50,23\% en la categoría de "mucho".

En cuanto a los valores medios (gráfico 3 siguiente), y respecto a la importancia en el trabajo, en general la media obtenida es superior al 3,2 de "sensibilidad medioambiental", llegando a la cota superior de 4,30 de "aplicación de conocimientos a la práctica", seguida del 4,16, en la "adaptación a nuevas situaciones" e "iniciativa y espíritu emprendedor" y el 4,08 de" liderazgo". Mientras, respecto al grado de preparación, las medias oscilan entre el 2,3 de "sensibilidad medioambiental", y el 3,2 de "búsqueda de información e investigación".

Según la información segmentada por submuestras, en general la valoración media de los varones es ligeramente superior a la de sus compañeras las féminas, en todas las competencias cuando se refiere a la importancia en el trabajo, y con la excepción de "capacidad para divulgar las cuestiones económicas" y "orientación al logro" en lo que respecta al grado de preparación en la Universidad. Además, en la segmentación por tener o no experiencia profesional, como regla, para el total de alumnos la valoración media del grado de desarrollo o preparación en la Universidad de las competencias es mayor cuando tienen experiencia, regularidad que también se observa en el caso de la importancia en el trabajo, a excepción de "motivación por la calidad", "aplicar conocimientos a la práctica" y "búsqueda de información e investigación".

\footnotetext{
${ }^{8}$ Véase las tablas del anexo para el listado de competencias analizadas
} 


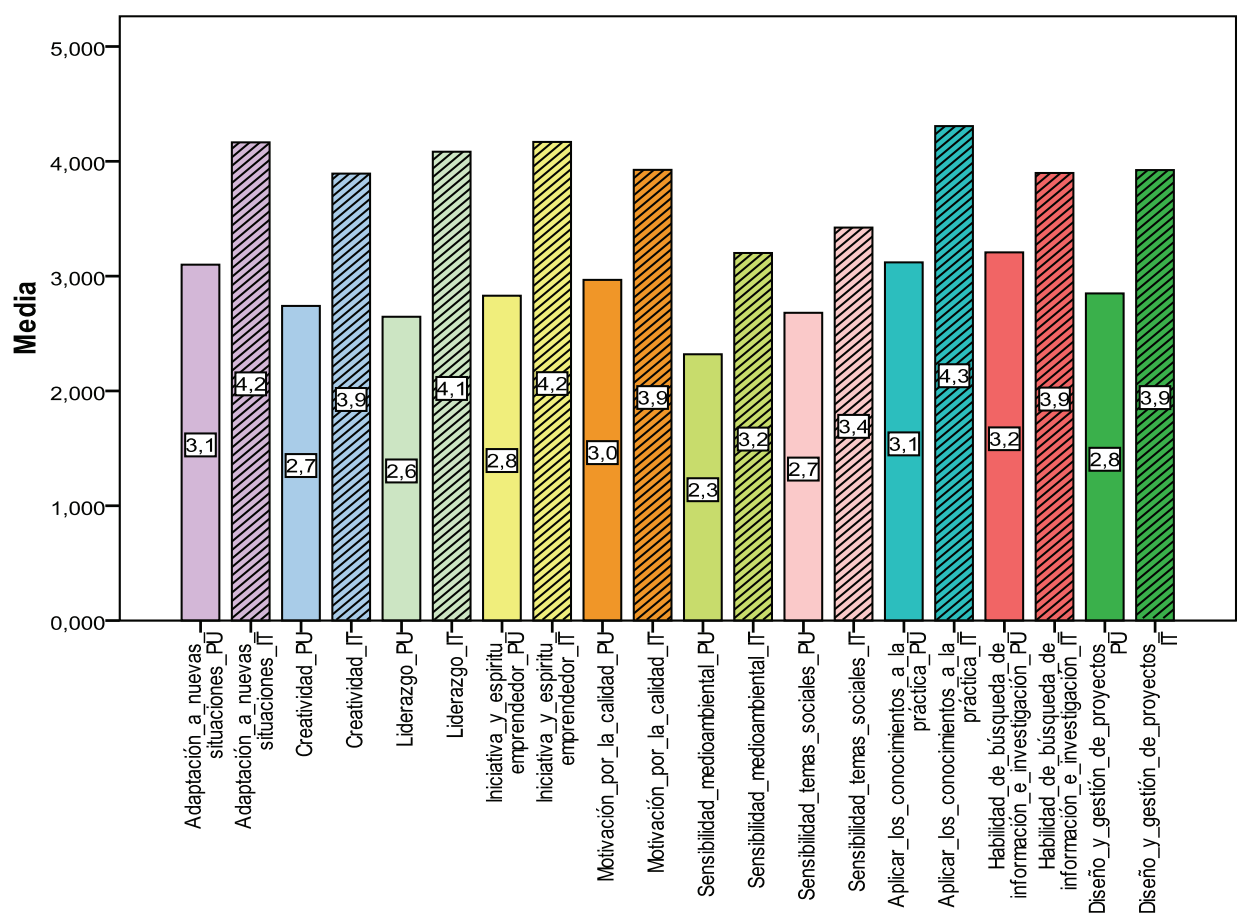

Gráfico 3: Valores medios Tercer Bloque de competencias. Preparación en la Universidad (PU) e Importancia en el trabajo (IT)

FUENTE: Encuesta de Necesidad de Desarrollo Competencial. Facultad de CCEE. Elaboración propia

\section{Cuarto Bloque de competencias 9}

El cuarto y último bloque de competencias que se presenta muestra que en general la opción más representativa cuando se pregunta por el grado de desarrollo o de formación en la Universidad es la de "regular", con porcentajes que oscilan entre el $27,25 \%$ de alumnos en "confianza en uno mismo" y el 40,32\% en "divulgación de las cuestiones económicas", con excepciones como "presentar en público productos, ideas o informes en público", "redactar informes o documentos" y "expresar ideas y defender derechos sin agredir", que acumulan mayoritariamente a los alumnos en la opción de "bastante", con porcentajes de alumnos que van del 32,88 al 29,28\%.

Respecto a la importancia que creen debe tener las distintas competencias en el entorno laboral, la opción más votada se reparte entre la de "bastante", con porcentajes que oscilan del 35,81\% en "autoevaluación emocional" al 42,79\% en "orientación al logro", y la de "mucho" que acumula mayoritariamente a los estudiantes con

\footnotetext{
${ }^{9}$ Véase las tablas del anexo para el listado de competencias analizadas
} 
porcentajes del $49,55 \%$ y el $46,85 \%$ respectivamente en las competencias de "confianza en uno mismo" y "expresar ideas y defender derechos sin agredir".

En cuanto a los valores medios, gráfico 4, y respecto a la importancia en el trabajo, la media obtenida es superior al 3,59 de "autoevaluación emocional", llegando a la cota superior de 4,2, en "redactar informes o documentos", "confianza en uno mismo" y "expresar ideas y defender derechos sin agredir". Mientras, respecto al grado de preparación, las medias oscilan entre el 2,46 de "negociación eficaz", y el 3,33 de "expresar ideas y defender derechos sin agredir".

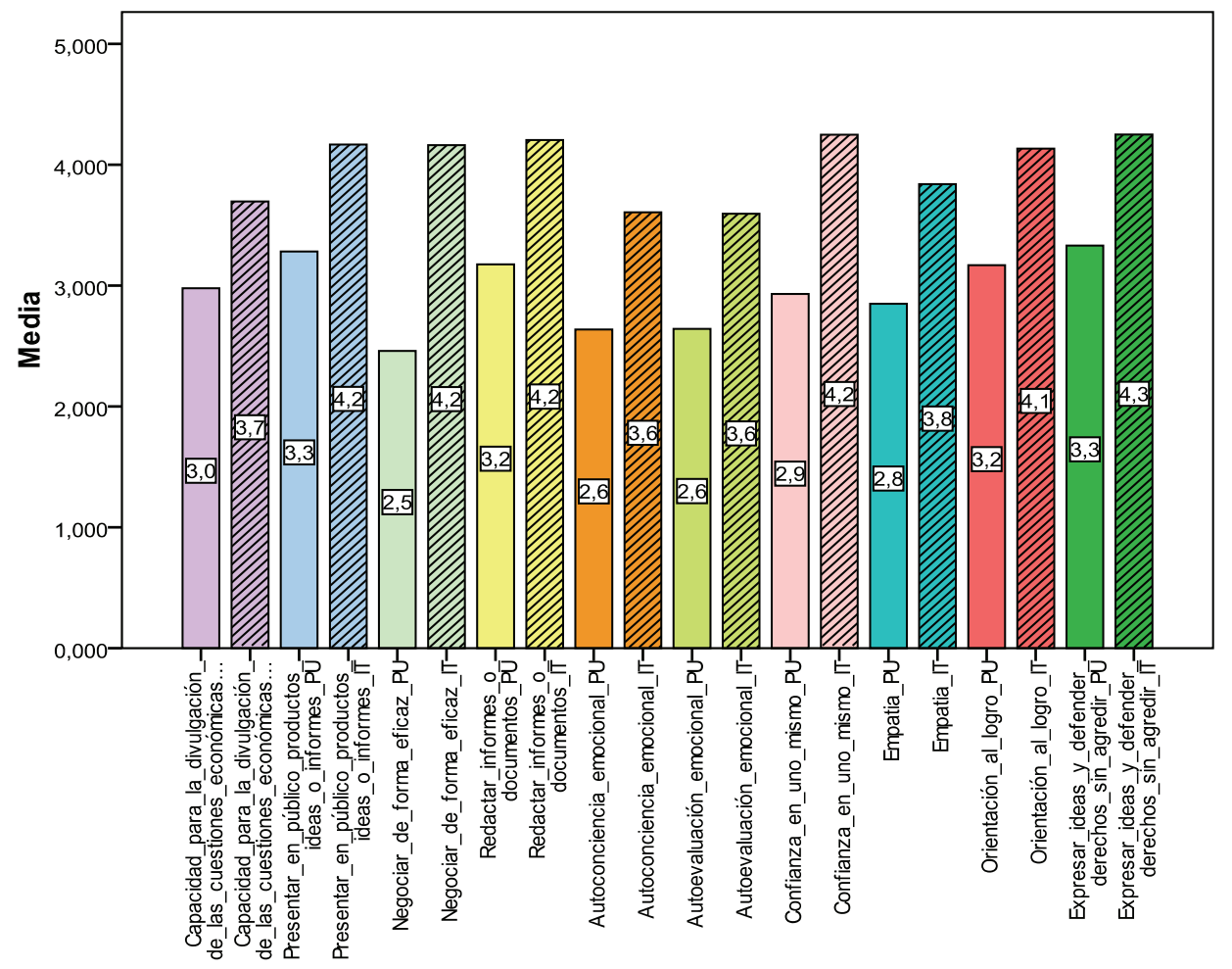

Gráfico 4: Valores medios Cuarto Bloque de competencias. Preparación en la Universidad (PU) e Importancia en el trabajo (IT)

FUENTE: Encuesta de Necesidad de Desarrollo Competencial. Facultad de CCEE. Elaboración propia.

Segmentando la información por submuestras, cabe destacar que en general la valoración media de los varones es ligeramente superior a la de sus compañeras las féminas, en todas las competencias cuando se refiere a la importancia en el trabajo, y con la excepción de "negociar de forma eficaz" y "la orientación al logro" en lo que respecta al grado de preparación en la Universidad. Además, al segmentar según la experiencia profesional, como regla y para el total de alumnos la valoración media, 
tanto del grado de desarrollo en la Universidad de las competencias como de la importancia en el trabajo, es mayor cuando los estudiantes aportan experiencia previa y solo destaca la excepción de "redactar informes o documento" y "la orientación al logro", ésta última referida a la importancia en el entorno laboral.

\section{Resumen de los resultados}

Para finalizar el análisis descriptivo, y a modo de resumen, se aportan algunas consideraciones para las segmentaciones de la muestra por sexo, según el curso que realicen, cuarto o quinto, y la licenciatura de Economía o de Administracción y Dirección de Empresas (tablas 3 y 4 del anexo).

Cabe destacar como norma general ya señalada previamente, y que se confirma con las distintas segmentaciones propuestas, que la valoración media de los estudiantes de la importancia que conceden a las distintas competencias en el trabajo es mayor que el grado de desarrollo competencial que declaran tener. Además, en la segmentación por curso en el que el alumno está matriculado, en general la valoración media del grado de desarrollo o preparación es mayor entre los que cursan quinto que entre los de cuarto, si bien para la importancia en el trabajo es al contrario.

Segmentando la muestra por licenciatura, se observa que en general la valoración media de los estudiantes que cursan LADE es ligeramente menor que la de los que cursan LECO tanto cuando la pregunta se refiere al grado de desarrollo o preparación universitaria, como a la importancia que perciben que debe tener en el trabajo. En este último caso, la relación señalada es una regularidad a excepción de las competencias "comunicación oral y escrita en lengua materna, búsqueda de información en diversas fuentes, compromiso ético en el trabajo, motivación por la calidad, divulgación de las ideas económicas y empatía".

\section{Conclusiones y valoración}

Es generalmente admitido entre el claustro universitario que el éxito de la acción docente no es sólo responsabilidad del alumno, ni se deriva únicamente del grado de esfuerzo de éste en el proceso de aprendizaje, sino que tanto las instituciones universitarias como los propios docentes tienen la responsabilidad social de facilitar una formación superior de calidad, que prepare a profesionales altamente cualificados con expectativas reales de inserción laboral, según los requerimientos cambiantes del mercado laboral. En este sentido, el marco actual de cambio metodológico en la Universidad como apuesta de futuro, debe comprometer a la Universidad en general, y a los docentes en particular, en la mejora de la calidad docente que dé respuesta a las necesidades de una sociedad vez más globalizada y que avanza hacia altas cotas de homogeneidad en el perfil de los egresados. Cada docente debe asumir que la mejora de la calidad en la educación superior pasa por "redefinir" la actividad del profesortutor, para lo cual es elemento básico un mejor y mayor conocimiento de la efectividad de la docencia impartida, en un proceso continuo de "reaprender" la práctica docente profesional "haciéndola". 
En este sentido, el trabajo trata de mostrar cómo cualquier docente debe acercar u orientar parte de su investigación a un mejor conocimiento de la efectividad de la acción docente, para así conocer los puntos fuertes y los débiles y generar con ello un proceso de retroalimentación que acerque la docencia al mundo profesional, a través de generar actividades de aprendizaje y académicas que reduzcan la brecha señalada entre el desarrollo competencial adquirido por un estudiante/egresado durante su etapa formativa universitaria y los requerimientos del entorno laboral. En definitiva, se trata de poner, porqué no, la investigación en didáctica al servicio de la docencia, en cualquier disciplina y área de conocimiento, para tener instrumentos de valoración sobre los diversos aspectos de la misma, tanto sobre efectividad docente, eficiencia de las instituciones, grados de absentismo, valoración de los docentes, o adecuación del desarrollo competencial.

\section{Referencias Bibliográficas}

IgLESIA E IONITA (2010), Percepción de la necesidad de un desarrollo competencial en los estudios de economía y administración de empresas. Documento de trabajo $\mathrm{n}^{\circ}$ 60, Junio 2010, Instituto de Análisis Industrial y Financiero. UCM.

IRIONDO (coord.), GALLEGO, GRANDAL, GRACIA, IGLESIA (2009a), Inserción Profesional de las principales licenciaturas de la UCM. Informe 2008. Ed. Ecobook.

IRIONDO (coord.), GALLEGO, GRANDAL, IGLESIA (2009b), La Inserción Profesional de los licenciados en Economía y Empresa de la UCM. Informe 2006. Ed. Ecobook.

\section{Correspondencia con la autora}

$\mathrm{M}^{\mathrm{a}}$ Covadonga de la Iglesia Villasol

Departamento de Fundamentos del Análisis Económico I

Universidad Complutense de Madrid

e-mail: $\underline{\text { civ@ccee.ucm.es }}$ 
Anexos






\begin{tabular}{|l|c|c|c|c|c|c|}
\hline \multicolumn{1}{|c|}{ Ciseño y gestión de proyectos } & 2,864 & 2,835 & 2,849 & 4,068 & 3,781 & 3,923 \\
\hline $\begin{array}{l}\text { Capacidad para la divulgación de las } \\
\text { cuestiones económicas }\end{array}$ & 2,909 & 3,045 & 2,977 & 3,836 & 3,558 & 3,696 \\
\hline $\begin{array}{l}\text { Presentar en público productos, ideas o } \\
\text { informes }\end{array}$ & 3,336 & 3,228 & 3,282 & 4,309 & 4,027 & 4,167 \\
\hline Negociar de forma eficaz & 2,659 & 2,263 & 2,459 & 4,273 & 4,054 & 4,162 \\
\hline Redactar informes o documentos & 3,227 & 3,125 & 3,176 & 4,382 & 4,031 & 4,205 \\
\hline Autoconciencia emocional & 2,686 & 2,589 & 2,637 & 3,850 & 3,366 & 3,606 \\
\hline Autoevaluación emocional & 2,714 & 2,571 & 2,642 & 3,818 & 3,375 & 3,595 \\
\hline Confianza en uno mismo & 2,945 & 2,915 & 2,930 & 4,414 & 4,085 & 4,248 \\
\hline $\begin{array}{l}\text { Empatía } \\
\text { Orientación al logro }\end{array}$ & 2,864 & 2,835 & 2,849 & 4,045 & 3,638 & 3,840 \\
\hline $\begin{array}{l}\text { Expresar ideas y defender derechos sin } \\
\text { agredir }\end{array}$ & 3,377 & 3,286 & 3,331 & 4,414 & 4,089 & 4,250 \\
\hline
\end{tabular}

Tabla 1. Media de la valoración del grado de preparación en la Universidad y la importancia en el trabajo

FUENTE: Encuesta de Necesidad de Desarrollo Competencial. Facultad de CCEE. Elaboración propia. 


\begin{tabular}{|c|c|c|c|c|}
\hline \multirow{2}{*}{$\begin{array}{c}\text { Competencia } \\
\text { Primer bloque }\end{array}$} & \multicolumn{2}{|c|}{$\begin{array}{c}\text { Preparación Universidad } \\
\text { Experiencia laboral } \\
\end{array}$} & \multicolumn{2}{|c|}{$\begin{array}{c}\text { Importancia Trabajo } \\
\text { Experiencia laboral } \\
\end{array}$} \\
\hline & SI & NO & SI & NO \\
\hline Pensamiento Analítico y Capacidad de sintesis & 3,409 & 3,376 & 3,948 & 3,945 \\
\hline Organización y Planificación & 3,461 & 3,276 & 4,273 & 4,283 \\
\hline Comunicación oral y escrita en lengua materna & 3,545 & 3,431 & 4,377 & 4,290 \\
\hline Comunicación oral y escrita en lengua extranjera & 2,305 & 2,076 & 4,299 & 4,193 \\
\hline Utilizar herramientas informáticas & 2,981 & 2,752 & 4,318 & 4,400 \\
\hline Búsqueda de información en diversas fuentes & 3,351 & 3,228 & 3,812 & 3,862 \\
\hline Resolución de conflictos o problemas & 3,013 & 2,752 & 4,084 & 4,193 \\
\hline Toma de decisiones & 3,084 & 2,821 & 4,292 & 4,310 \\
\hline Conocimientos de otras áreas o disciplinas & 2,818 & 2,748 & 3,513 & 3,448 \\
\hline Adquirir con rapidez nuevos conocimientos & 3,357 & 3,310 & 3,877 & 4,028 \\
\hline Segundo bloque & SI & NO & SI & NO \\
\hline Capacidad para hacerte entender & 3,260 & 3,169 & 4,253 & 4,217 \\
\hline Trabajar en equipo de carácter multidisciplinar & 3,314 & 3,276 & 4,182 & 4,266 \\
\hline Trabajo en un Contexto internacional & 2,526 & 2,303 & 3,877 & 3,686 \\
\hline $\begin{array}{l}\text { Habilidad en relaciones personales y } \\
\text { Comunicación interpersonal }\end{array}$ & 3,156 & 2,917 & 4,149 & 4,055 \\
\hline Trabajar en Entornos diversos y multiculturales & 2,903 & 2,531 & 3,792 & 3,655 \\
\hline Capacidad Crítica y autocrítica & 3,078 & 2,928 & 3,903 & 3,821 \\
\hline Compromiso ético en el trabajo & 3,045 & 2,852 & 3,994 & 3,838 \\
\hline Rendir bajo presión & 3,532 & 3,390 & 4,117 & 4,214 \\
\hline Usar el tiempo de forma efectiva & 3,266 & 3,128 & 4,325 & 4,168 \\
\hline Aprendizaje autónomo & 3,506 & 3,572 & 3,831 & 4,290 \\
\hline Tercer bloque & SI & NO & SI & NO \\
\hline Adaptación a nuevas situaciones & 3,149 & 3,072 & 4,201 & 4,145 \\
\hline Creatividad & 2,838 & 2,690 & 3,981 & 3,845 \\
\hline Liderazgo & 2,727 & 2,603 & 4,123 & 4,062 \\
\hline $\begin{array}{l}\text { Iniciativa y espíritu emprendedor : encontrar } \\
\text { nuevas ideas y soluciones }\end{array}$ & 2,916 & 2,783 & 4,240 & 4,131 \\
\hline Motivación por la calidad & 3,039 & 2,928 & 3,870 & 3,955 \\
\hline Sensibilidad medioambiental & 2,403 & 2,276 & 3,318 & 3,138 \\
\hline Sensibilidad temas sociales & 2,831 & 2,600 & 3,623 & 3,317 \\
\hline Aplicar los conocimientos a la práctica & 3,240 & 3,055 & 4,279 & 4,321 \\
\hline $\begin{array}{l}\text { Habilidad de búsqueda de información e } \\
\text { investigación }\end{array}$ & 3,208 & 3,207 & 3,896 & 3,900 \\
\hline Diseño y gestión de proyectos & 2,929 & 2,807 & 3,955 & 3,907 \\
\hline Cuarto bloque & SI & NO & SI & NO \\
\hline $\begin{array}{l}\text { Capacidad para la divulgación de las cuestiones } \\
\text { económicas }\end{array}$ & 3,078 & 2,924 & 3,740 & 3,672 \\
\hline Presentar en público productos, ideas o informes & 3,325 & 3,259 & 4,221 & 4,138 \\
\hline
\end{tabular}




\begin{tabular}{|l|c|c|c|c|} 
Negociar de forma eficaz & 2,565 & 2,403 & 4,195 & 4,145 \\
\hline Redactar informes o documentos & 3,169 & 3,179 & 4,175 & 4,221 \\
\hline Autoconciencia emocional & 2,786 & 2,559 & 3,656 & 3,579 \\
\hline Autoevaluación emocional & 2,838 & 2,538 & 3,623 & 3,579 \\
\hline Confianza en uno mismo & 3,071 & 2,855 & 4,286 & 4,228 \\
\hline Empatía & 2,929 & 2,807 & 3,857 & 3,831 \\
\hline Orientación al logro & 3,175 & 3,166 & 4,071 & 4,166 \\
\hline Expresar ideas y defender derechos sin agredir & 3,558 & 3,210 & 4,344 & 4,200 \\
\hline
\end{tabular}

Tabla 2. Media de la valoración del grado de preparación en la Universidad y la importancia en el trabajo,

FUENTE: Encuesta de Necesidad de Desarrollo Competencial. Facultad de CCEE. Elaboración propia. 


\begin{tabular}{|c|c|c|c|c|}
\hline \multirow{2}{*}{ Competencias } & \multicolumn{2}{|c|}{$\begin{array}{c}\text { CUARTO curso } \\
\text { Experiencia laboral }\end{array}$} & \multicolumn{2}{|c|}{$\begin{array}{c}\text { QUINTO curso } \\
\text { Experiencia laboral }\end{array}$} \\
\hline & $\begin{array}{l}\text { Preparación } \\
\text { Universidad }\end{array}$ & $\begin{array}{c}\text { Importancia } \\
\text { trabajo }\end{array}$ & $\begin{array}{l}\text { Preparación } \\
\text { Universidad }\end{array}$ & $\begin{array}{c}\text { Importancia } \\
\text { trabajo }\end{array}$ \\
\hline Primer bloque & Total & Total & Total & Total \\
\hline $\begin{array}{l}\text { Pensamiento Analitico y } \\
\text { Capacidad de sintesis }\end{array}$ & 3,349 & 3,949 & 3,439 & 3,942 \\
\hline Organización y Planificación & 3,275 & 4,263 & 3,429 & 4,302 \\
\hline $\begin{array}{l}\text { Comunicación oral y escrita en } \\
\text { lengua materna }\end{array}$ & 3,455 & 4,333 & 3,492 & 4,302 \\
\hline $\begin{array}{l}\text { Comunicación oral y escrita en } \\
\text { lengua extranjera }\end{array}$ & 2,086 & 4,224 & 2,249 & 4,238 \\
\hline Utilizar herramientas informáticas & 2,757 & 4,427 & 2,931 & 4,296 \\
\hline $\begin{array}{l}\text { Búsqueda de información en } \\
\text { diversas fuentes }\end{array}$ & 3,216 & 3,929 & 3,344 & 3,730 \\
\hline $\begin{array}{l}\text { Resolución de conflictos } o \\
\text { problemas }\end{array}$ & 2,800 & 4,200 & 2,899 & 4,095 \\
\hline Toma de decisiones & 2,937 & 4,322 & 2,878 & 4,280 \\
\hline $\begin{array}{l}\text { Conocimientos de otras áreas o } \\
\text { disciplinas }\end{array}$ & 2,753 & 3,549 & 2,799 & 3,365 \\
\hline $\begin{array}{l}\text { Adquirir con rapidez nuevos } \\
\text { conocimientos }\end{array}$ & 3,353 & 4,043 & 3,291 & 3,884 \\
\hline Segundo bloque & Total & Total & Total & Total \\
\hline Capacidad para hacerte entender & 3,224 & 4,278 & 3,169 & 4,164 \\
\hline $\begin{array}{l}\text { Trabajar en equipo de carácter } \\
\text { multidisciplinar }\end{array}$ & 3,310 & 4,298 & 3,261 & 4,153 \\
\hline $\begin{array}{l}\text { Trabajo en un Contexto } \\
\text { internacional }\end{array}$ & 2,365 & 3,780 & 2,402 & 3,714 \\
\hline $\begin{array}{l}\text { Habilidad en relaciones personales } \\
\text { y Comunicación interpersonal }\end{array}$ & 3,008 & 4,145 & 2,989 & 4,011 \\
\hline $\begin{array}{l}\text { Trabajar en Entornos diversos y } \\
\text { multiculturales }\end{array}$ & 2,620 & 3,686 & 2,714 & 3,725 \\
\hline Capacidad Crítica y autocrítica & 2,957 & 3,843 & 3,011 & 3,857 \\
\hline Compromiso ético en el trabajo & 2,898 & 3,910 & 2,947 & 3,868 \\
\hline Rendir bajo presión & 3,557 & 4,263 & 3,280 & 4,069 \\
\hline Usar el tiempo de forma efectiva & 3,122 & 4,349 & 3,249 & 4,238 \\
\hline Aprendizaje autónomo & 3,600 & 3,871 & 3,481 & 3,862 \\
\hline Tercer bloque & Total & Total & Total & Total \\
\hline Adaptación a nuevas situaciones & 3,098 & 4,192 & 3,101 & 4,127 \\
\hline Creatividad & 2,804 & 3,878 & 2,656 & 3,910 \\
\hline Liderazgo & 2,639 & 4,043 & 2,656 & 4,138 \\
\hline $\begin{array}{l}\text { Iniciativa y espiritu emprendedor: } \\
\text { encontrar nuevas ideas y } \\
\text { soluciones }\end{array}$ & 2,859 & 4,165 & 2,788 & 4,175 \\
\hline Motivación por la calidad & 2,980 & 3,988 & 2,947 & 3,841 \\
\hline Sensibilidad medioambiental & 2,329 & 3,216 & 2,307 & 3,180 \\
\hline Sensibilidad temas sociales & 2,722 & 3,400 & 2,624 & 3,455 \\
\hline $\begin{array}{l}\text { Aplicar los conocimientos a la } \\
\text { práctica }\end{array}$ & 3,125 & 4,271 & 3,111 & 4,354 \\
\hline
\end{tabular}




\begin{tabular}{|l|c|c|c|c|}
\begin{tabular}{|l|l|l|} 
Habilidad de búsqueda de \\
información e investigación
\end{tabular} & 3,200 & 3,906 & 3,217 & 3,889 \\
\hline Diseño y gestión de proyectos & 2,839 & 3,965 & 2,862 & 3,868 \\
\hline \multicolumn{1}{|c|}{ Cuarto bloque } & Total & Total & Total & Total \\
\hline $\begin{array}{l}\text { Capacidad para la divulgación de } \\
\text { las cuestiones económicas }\end{array}$ & 2,961 & 3,682 & 3,000 & 3,714 \\
\hline $\begin{array}{l}\text { Presentar en público productos, } \\
\text { ideas o informes }\end{array}$ & 3,373 & 4,165 & 3,159 & 4,169 \\
\hline Negociar de forma eficaz & 2,467 & 4,157 & 2,450 & 4,169 \\
\hline Redactar informes o documentos & 3,259 & 4,227 & 3,063 & 4,175 \\
\hline Autoconciencia emocional & 2,671 & 3,600 & 2,593 & 3,614 \\
\hline Autoevaluación emocional & 2,635 & 3,600 & 2,651 & 3,587 \\
\hline Confianza en uno mismo & 2,898 & 4,286 & 2,974 & 4,196 \\
\hline Empatía & 2,847 & 3,878 & 2,852 & 3,788 \\
\hline Orientación al logro & 3,161 & 4,192 & 3,180 & 4,053 \\
\hline $\begin{array}{l}\text { Expresar ideas y defender } \\
\text { derechos sin agredir }\end{array}$ & 3,322 & 4,247 & 3,344 & 4,254 \\
\hline
\end{tabular}

Tabla 3. Media de la valoración del grado de preparación en la Universidad y la importancia en el trabajo, por curso

FUENTE: Encuesta de Necesidad de Desarrollo Competencial. Facultad de CCEE. Elaboración propia. 


\begin{tabular}{|c|c|c|c|c|}
\hline \multirow{3}{*}{ Competencias } & \multirow{2}{*}{\multicolumn{2}{|c|}{$\begin{array}{c}\text { LECO } \\
\text { Experiencia laboral }\end{array}$}} & \multirow{2}{*}{\multicolumn{2}{|c|}{$\begin{array}{c}\text { LADE } \\
\text { Experiencia laboral }\end{array}$}} \\
\hline & & & & \\
\hline & $\begin{array}{l}\text { Preparación } \\
\text { Universidad }\end{array}$ & $\begin{array}{c}\text { Importancia } \\
\text { trabajo }\end{array}$ & $\begin{array}{l}\text { Preparación } \\
\text { Universidad }\end{array}$ & $\begin{array}{c}\text { Importancia } \\
\text { trabajo }\end{array}$ \\
\hline Primer bloque & Total & Total & Total & Total \\
\hline $\begin{array}{l}\text { Pensamiento Analítico y } \\
\text { Capacidad de síntesis }\end{array}$ & 3,299 & 3,966 & 3,556 & 3,908 \\
\hline Organización y Planificación & 3,364 & 4,313 & 3,294 & 4,216 \\
\hline $\begin{array}{l}\text { Comunicación oral y escrita en } \\
\text { lengua materna }\end{array}$ & 3,436 & 4,299 & 3,536 & 4,359 \\
\hline $\begin{array}{l}\text { Comunicación oral y escrita en } \\
\text { lengua extranjera }\end{array}$ & 2,093 & 4,254 & 2,275 & 4,183 \\
\hline Utilizar herramientas informáticas & 2,711 & 4,433 & 3,059 & 4,255 \\
\hline $\begin{array}{l}\text { Búsqueda de información en } \\
\text { diversas fuentes }\end{array}$ & 3,158 & 3,842 & 3,484 & 3,850 \\
\hline $\begin{array}{l}\text { Resolución de conflictos o } \\
\text { problemas }\end{array}$ & 2,866 & 4,203 & 2,797 & 4,065 \\
\hline Toma de decisiones & 2,983 & 4,371 & 2,778 & 4,176 \\
\hline $\begin{array}{l}\text { Conocimientos de otras áreas o } \\
\text { disciplinas }\end{array}$ & 2,814 & 3,533 & 2,693 & 3,353 \\
\hline $\begin{array}{l}\text { Adquirir con rapidez nuevos } \\
\text { conocimientos }\end{array}$ & 3,285 & 3,986 & 3,405 & 3,954 \\
\hline Segundo bloque & Total & Total & Total & Total \\
\hline Capacidad para hacerte entender & 3,148 & 4,234 & 3,301 & 4,222 \\
\hline $\begin{array}{l}\text { Trabajar en equipo de carácter } \\
\text { multidisciplinar }\end{array}$ & 3,390 & 4,271 & 3,098 & 4,170 \\
\hline $\begin{array}{l}\text { Trabajo en un Contexto } \\
\text { internacional }\end{array}$ & 2,306 & 3,777 & 2,523 & 3,706 \\
\hline $\begin{array}{l}\text { Habilidad en relaciones } \\
\text { personales y Comunicación } \\
\text { interpersonal }\end{array}$ & 2,986 & 4,137 & 3,026 & 3,993 \\
\hline $\begin{array}{l}\text { Trabajar en Entornos diversos y } \\
\text { multiculturales }\end{array}$ & 2,639 & 3,735 & 2,699 & 3,641 \\
\hline Capacidad Crítica y autocrítica & 2,993 & 3,904 & 2,954 & 3,745 \\
\hline Compromiso ético en el trabajo & 2,952 & 3,887 & 2,856 & 3,902 \\
\hline Rendir bajo presión & 3,460 & 4,237 & 3,399 & 4,072 \\
\hline Usar el tiempo de forma efectiva & 3,189 & 4,320 & 3,150 & 4,268 \\
\hline Aprendizaje autónomo & 3,553 & 3,876 & 3,542 & 3,850 \\
\hline Tercer bloque & Total & Total & Total & Total \\
\hline Adaptación a nuevas situaciones & 3,093 & 4,213 & 3,111 & 4,072 \\
\hline Creatividad & 2,801 & 3,962 & 2,627 & 3,758 \\
\hline Liderazgo & 2,715 & 4,148 & 2,516 & 3,961 \\
\hline $\begin{array}{l}\text { Iniciativa y espíritu emprendedor: } \\
\text { encontrar nuevas ideas y } \\
\text { soluciones }\end{array}$ & 2,863 & 4,192 & 2,765 & 4,124 \\
\hline Motivación por la calidad & 2,938 & 3,911 & 3,020 & 3,954 \\
\hline Sensibilidad medioambiental & 2,296 & 3,237 & 2,366 & 3,131 \\
\hline
\end{tabular}




\begin{tabular}{|l|l|l|l|l|} 
Sensibilidad temas sociales & 2,601 & 3,443 & 2,830 & 3,386 \\
\hline $\begin{array}{l}\text { Aplicar los conocimientos a la } \\
\text { práctica }\end{array}$ & 3,141 & 4,378 & 3,078 & 4,170 \\
\hline $\begin{array}{l}\text { Habilidad de búsqueda de } \\
\text { información e investigación }\end{array}$ & 3,168 & 3,945 & 3,281 & 3,810 \\
\hline Diseño y gestión de proyectos & 2,900 & 3,962 & 2,752 & 3,850 \\
\hline \multicolumn{1}{|c|}{ Cuarto bloque } & Total & Total & Total & Total \\
\hline $\begin{array}{l}\text { Capacidad para la divulgación de } \\
\text { las cuestiones económicas }\end{array}$ & 2,890 & 3,660 & 3,144 & 3,765 \\
\hline $\begin{array}{l}\text { Presentar en público productos, } \\
\text { ideas o informes }\end{array}$ & 3,381 & 4,220 & 3,092 & 4,065 \\
\hline Negociar de forma eficaz & 2,481 & 4,168 & 2,418 & 4,150 \\
\hline Redactar informes o documentos & 3,134 & 4,216 & 3,255 & 4,183 \\
\hline Autoconciencia emocional & 2,680 & 3,622 & 2,556 & 3,575 \\
\hline Autoevaluación emocional & 2,660 & 3,601 & 2,608 & 3,582 \\
\hline Confianza en uno mismo & 2,931 & 4,296 & 2,928 & 4,157 \\
\hline Empatía & 2,869 & 3,838 & 2,810 & 3,843 \\
\hline Orientación al logro & 3,189 & 4,179 & 3,131 & 4,046 \\
\hline $\begin{array}{l}\text { Expresar ideas y defender } \\
\text { derechos sin agredir }\end{array}$ & 3,313 & 4,289 & 3,366 & 4,176 \\
\hline
\end{tabular}

Tabla 4. Media de la valoración del grado de preparación en la Universidad y la importancia en el trabajo, por titulación

FUENTE: Encuesta de Necesidad de Desarrollo Competencial. Facultad de CCEE. Elaboración propia. 


\begin{tabular}{|c|c|c|c|c|c|c|c|c|c|c|}
\hline \multirow{2}{*}{$\begin{array}{c}\text { Competencias } \\
\text { Primer } \\
\text { bloque } \\
\end{array}$} & \multicolumn{5}{|c|}{ Preparación en la Universidad } & \multicolumn{5}{|c|}{ Importancia en el trabajo } \\
\hline & $\begin{array}{c}1 \\
\text { Nada }\end{array}$ & $\begin{array}{c}2 \\
\text { Poco }\end{array}$ & $\begin{array}{c}3 \\
\text { Regular }\end{array}$ & \begin{tabular}{|c|}
4 \\
Bastante
\end{tabular} & \begin{tabular}{|c|}
5 \\
Mucho
\end{tabular} & $\begin{array}{c}1 \\
\text { Nada }\end{array}$ & $\begin{array}{c}2 \\
\text { Poco }\end{array}$ & $\begin{array}{c}3 \\
\text { Regular }\end{array}$ & \begin{tabular}{|c|}
4 \\
Bastante
\end{tabular} & $\begin{array}{c}5 \\
\text { Mucho }\end{array}$ \\
\hline & 2,25 & 12,16 & 38,74 & 38,29 & 8,56 & 0,90 & 4,05 & 20,50 & 48,65 & 25,90 \\
\hline & 2,93 & 22,30 & 28,15 & 31,08 & 15,54 & 0,90 & 1,80 & 9,91 & 43,24 & 44,14 \\
\hline & 4,05 & 13,51 & 31,98 & 31,98 & 18,24 & 0,45 & 1,80 & 12,61 & 35,59 & 49,55 \\
\hline & 38,29 & 28,60 & 17,79 & 9,91 & 5,41 & 2,25 & 3,15 & 14,86 & 28,83 & 50,90 \\
\hline & 11,71 & 29,05 & 31,76 & 19,37 & 8,11 & 0,68 & 1,13 & 8,78 & 39,19 & 50,23 \\
\hline & 4,05 & 19,59 & 31,76 & 34,46 & 10,14 & 0,68 & 4,50 & 26,58 & 46,17 & 22,07 \\
\hline & 10,36 & 27,03 & 34,91 & 23,42 & 4,28 & 1,13 & 3,15 & 15,09 & 40,32 & 40,32 \\
\hline & 10,81 & 21,40 & 39,41 & 22,52 & 5,86 & 0,45 & 2,93 & 10,36 & 38,29 & 47,97 \\
\hline & 13,29 & 28,60 & 30,41 & 22,97 & 4,73 & 2,03 & 11,49 & 36,49 & 37,39 & 12,61 \\
\hline & 3,60 & 15,99 & 36,26 & 32,43 & 11,71 & 0,23 & 3,83 & 22,30 & 45,50 & 28,15 \\
\hline \multirow[t]{11}{*}{$\begin{array}{c}\text { Segundo } \\
\text { Bloque }\end{array}$} & Nada & Poco & Regular & Bastante & Mucho & Nada & Poco & Regular & Bastante & Mucho \\
\hline & 4,73 & 18,69 & 36,94 & 31,08 & 8,56 & 0,23 & 2,93 & 14,86 & 37,61 & 44,37 \\
\hline & 5,64 & 15,58 & 33,18 & 35,44 & 10,16 & 0,45 & 2,25 & 13,74 & 40,32 & 43,24 \\
\hline & 26,35 & 31,08 & 24,77 & 13,74 & 4,05 & 3,60 & 6,31 & 25,23 & 40,99 & 23,87 \\
\hline & 7,88 & 22,07 & 38,74 & 24,77 & 6,53 & 0,90 & 2,25 & 17,12 & 46,62 & 33,11 \\
\hline & 18,24 & 29,95 & 25,90 & 19,37 & 6,53 & 3,38 & 8,33 & 27,48 & 36,26 & 24,55 \\
\hline & 6,98 & 25,00 & 37,39 & 24,32 & 6,31 & 1,13 & 5,41 & 25,68 & 43,02 & 24,77 \\
\hline & 13,74 & 20,27 & 36,04 & 20,27 & 9,68 & 3,83 & 4,95 & 22,07 & 36,49 & 32,66 \\
\hline & 7,66 & 11,71 & 29,73 & 30,86 & 20,05 & 1,58 & 3,15 & 14,64 & 36,94 & 43,69 \\
\hline & 9,91 & 17,79 & 33,11 & 23,20 & 15,99 & 0,90 & 2,25 & 9,68 & 40,09 & 47,07 \\
\hline & 3,83 & 11,49 & 27,70 & 39,86 & 17,12 & 1,13 & 3,83 & 25,45 & 46,40 & 23,20 \\
\hline \multirow[t]{11}{*}{$\begin{array}{l}\text { Tercer } \\
\text { Bloque }\end{array}$} & Nada & Poco & Regular & Bastante & Mucho & Nada & Poco & Regular & Bastante & Mucho \\
\hline & 6,08 & 20,05 & 39,41 & 26,80 & 7,66 & 0,23 & 2,70 & 15,09 & 44,37 & 37,61 \\
\hline & 14,64 & 25,68 & 35,59 & 19,14 & 4,95 & 1,80 & 5,63 & 20,72 & 45,27 & 26,58 \\
\hline & 15,77 & 31,31 & 31,76 & 14,86 & 6,31 & 1,13 & 3,60 & 18,24 & 39,86 & 37,16 \\
\hline & 10,36 & 28,38 & 33,78 & 22,97 & 4,50 & 0,90 & 2,70 & 15,32 & 40,77 & 40,32 \\
\hline & 9,68 & 22,30 & 36,49 & 24,77 & 6,76 & 0,45 & 4,95 & 25,23 & 40,32 & 29,05 \\
\hline & 31,53 & 28,60 & 21,40 & 13,29 & 5,18 & 7,66 & 20,27 & 30,18 & 28,15 & 13,74 \\
\hline & 18,02 & 27,03 & 30,18 & 18,47 & 6,31 & 4,50 & 15,32 & 30,63 & 32,43 & 17,12 \\
\hline & 9,68 & 18,02 & 33,56 & 28,15 & 10,59 & 0,68 & 1,58 & 14,41 & 33,11 & 50,23 \\
\hline & 5,86 & 18,92 & 31,53 & 36,04 & 7,66 & 0,90 & 3,60 & 23,42 & 48,87 & 23,20 \\
\hline & 11,71 & 23,20 & 38,29 & 22,07 & 4,73 & 1,35 & 4,73 & 20,50 & 47,07 & 26,35 \\
\hline \multirow[t]{8}{*}{$\begin{array}{l}\text { Cuarto } \\
\text { Bloque }\end{array}$} & Nada & Poco & Regular & Bastante & Mucho & Nada & Poco & Regular & Bastante & Mucho \\
\hline & 7,88 & 21,85 & 40,32 & 24,55 & 5,41 & 1,13 & 6,08 & 34,01 & 39,64 & 19,14 \\
\hline & 6,08 & 17,34 & 31,31 & 32,88 & 12,39 & 0,90 & 3,15 & 18,69 & 32,88 & 44,37 \\
\hline & 26,13 & 26,13 & 27,48 & 16,22 & 4,05 & 1,35 & 3,38 & 12,84 & 42,57 & 39,86 \\
\hline & 9,01 & 18,02 & 30,63 & 31,08 & 11,26 & 1,13 & 3,60 & 11,71 & 40,77 & 42,79 \\
\hline & 19,59 & 25,00 & 32,66 & 17,57 & 5,18 & 3,60 & 6,08 & 32,66 & 41,44 & 16,22 \\
\hline & 19,14 & 26,35 & 29,73 & 20,72 & 4,05 & 3,83 & 6,98 & 34,23 & 35,81 & 19,14 \\
\hline & 15,54 & 22,07 & 27,25 & 24,10 & 11,04 & 1,35 & 4,05 & 12,61 & 32,43 & 49,55 \\
\hline
\end{tabular}




\begin{tabular}{|c|c|c|c|c|c|c|c|c|c|c|}
\cline { 3 - 11 } & 13,96 & 22,52 & 33,78 & 24,10 & 5,63 & 2,03 & 6,08 & 26,80 & 36,04 & 29,05 \\
\hline & 8,11 & 17,57 & 32,66 & 32,66 & 9,01 & 0,90 & 2,48 & 16,44 & 42,79 & 37,39 \\
\hline & 8,33 & 15,99 & 28,15 & 29,28 & 18,24 & 1,35 & 1,80 & 14,19 & 35,81 & 46,85 \\
\hline
\end{tabular}

Tabla 5. Distribución de frecuencias de las competencias, por grado de preparación en la Universidad e importancia en el trabajo

FUENTE: Encuesta de Necesidad de Desarrollo Competencial. Facultad de CCEE. Elaboración propia. 